\title{
Bericht über die Sitzung der Moskauer augenärztlichen
}

\section{Gesellschaft}

vom 24. Februar (9. März) 1909

Fr. Dr. Jacub demonstriert einen Kranken mit sogenannter Blut-tinktion der Hornhaut, einer Veränderung, die schon öfters zu Verwechs-lungen mit der in die vordere Rammer luxierten Linse führte und so den Anlass zu operativen Eingriffen gab. In diesem Falle war eine Verwechslung wegen sehr beträchtlicher Tiefe der Kammer und vollständig klarer Beschaffenheit des Kammerwassers ausgeschlossen. Aetiologie - ein Trauma.

Dr. Snegire”. demonstriert einen 49 jährígen Mann mit Epithelkrebs-rezidiv am Limbus corneae. Der Kranke wurde von dem Vortragenden schon 4 mal operiert. Das erste Mai vor 6 Jahren, das letzte Mai vor Jahresfrist. Jedesmal zeigte sich das Rezidiv in Form einer kleinen, halbdurchsichtigen, an eine Phlyctäne erinnernden Geschwlust an der corneo-skleralen Grenze und wurde von starken Reizerscheinungen und Trübungen der Hornhaut hegleitet. Operation bestand in der Abkratzung der Geschwulst und Kauteri-sation des Limbus. Die Erkrankung bleibt stets lokal, die Funktion des Auges ungestört. Es besteht deshalb keine Indikation zur Enukleation.

Als erster Punkt der Tagesordnung folgt dann der Vortrag des Herrn Dr. Rochanitzin über Rundzellensarkome der Bindehaut. Vortr. macht darauf aufmerksam, dass die

Konjunktivalsarkome im ahgemeinen selten vorkommen, die Rundzellensarkome aber eine noch grössere Seltenheit darstellen und sich besonders durch ihre Bösartigkeit auszeichnen. Nach einem kurzen Ueberblick über die bis jetzt veröffentlichten Fälle von Konjunktivalsarkomberichtet er von zwei weiteren, die in der Moskauer Augen-klinik beobachtet wurden.

Der 1. Fall betrifft ein 7 jähriges Mädchen, bei dem sich die Geschwulst a us der Uebergangsfalte des linken oberen Lides vor 9 Monaten zu ent-wiekeln begann. Allmählich griff die Neubildung auf beide Fornices, die Karunkel und die halbmondförmige Falte über. Sie war schmerzlos, von weicher, elastischer Konsistenz, nicht mit der bedeckenden Haiit verwachsen und breitete sich diffus in dem umgebenden Gewebe aus. Die mikroskopische Untersuchung eines exzidierten Stückes zeigte, dass es sich um ein unpig-mentiertes Rundzellensarkom mit starker Einwucherung in die Gefässe handelt. Der Fall wurde als inoperabel angesehen, und die Kranke entzog sich weiterer Beobachtung.

2. Fall betrifft ein sehr vorgeschrittenes Stadium der Erkrankung. üer 68 jährige Mann bemerkte die ersten Anzeichen einer Geschwulst vor 3 Jahren. Sie entwickelte sich nacli seiner Angabe gleichzeitig auf beiden Augen. Trotz 3 maliger Operation auf dem Lande (1 mal links und 2 mal rechts) weiteres Fortschreiten. Die Untersuchung ergab, dass die Geschwulst beiderseits von der Conjunctiva bulbi ausgeht und sich sehr tief in die Orbita hinein ausbreitet. Das rechte Auge ist nach aussen oben, das linke nach innen oben verschoben, beide sind in ihrer Beweglichkeit beschränkt. Mikroskopisch handelte es sich auch hier um ein Rundzellensarkom mit starker Beteiligung der Gefässwände. Der Kranke wurde dem Institut für Ivrebsforschung überwiesen, verliess es aber in kurzer Zeit. 
Moskauer augenärztliche Gesellschaft.

371

Sodann spricht Dr. Oãinzow über einen Fall von RankenneilГom des oberen Lides, das bei einem 18 jährigen Kranken sich vor etwa 6 Jahren auf dem rechten Auge zu entwickeln begann. Die Geschwulst vergrösserte sich allraählich, verursachte dem Kranken keine Beschwerden, ausser einer ziemlich beträchtlichen Entstellung und geringgradiger Sehstörung, die davon abhing, dass die nasale Halite des von der Geschwulst ergriffenen Oberlides die Augenspalte beinahe vollständig bedeckte und nur den äusseren Teil derselben frei liess. Exstirpation. Glatte Heilung. Die anatomische Untersuchung ergab ein typisches Rankenneurom mit hauptsächlicher Beteiligung des Perineuriums und beginnender schleimiger Degeneration. Die Nervenfasern waren zumeist degeneriert, ihre Markscheide mit zahl-reichen Lantermannschen Einschnürungen. An der Hand der 60 Fälle um-fassenden Literatur gibt ferner der Vortragende das klinisch $\theta$ und anatomische Bild der Rankenneurome und spricht von der Kombination derselben mit Hydrophthalmus. Das Zusammentreffen dieser beiden Erkrankungen wurde von einigen Autoren in ursächlichen Zusammenhang gebracht. Nach der Ansicht des Vortragenden kann ein solcher Zusammenhang zugelassen werden, aber nur für Fälle, wo auch Ciliarnerven fibromatös entartet sind, wo also der Hydrophthalmus als Folge der Neurofibromatose anzusehen ist. Er kann sich aber nicht der Ansicht von v. Michel anschliessen, der beid $\theta$ Erkrankungen als Aeusserungen derselben Ursache,nämlich einer angeborenen fehlerhaften Entwicklung des Mesenchyms und in diesem Sinne den ein-seitigen Hydrophthalmus ohne andere Zeichen der Fibromatose als einzigen. Ausdruck derselben betrachtet.

Dr. Odínzon demonstriert weiter mikroskopische Präparate eines Linsen- und Zonulacoloboms. Die Präparate entstammen einem Schweins-auge. Die Linse war in ihrer unteren Peripherie von einer bogenförmigen Linie begrenzt. Die Zonula zeigte einen dreieckigen, 3-4 mm messenden Defekt. Als Ursache cles Coloboms wurde ein Bindegewebszug ermittelt, der sich auf der Pars plana Corporis ciliaris auf dem Pigmentepithel, das auch einen kleinen Defekt zeigte, befand und sich durch das ganze Colobomgebiet erstreckte. Der erwähnte Zug stellt wahrscheinlich einen abgeschnürten Rest vom Mesodermgewebe, welches im Fötalleben die Spalte der sekundären Augenblase ausfüllt, dar. Die Linse selbst zeigte auch eigentümliche Ver-änderungen, nämlich im Colobomgebiete richtete sich ihre Kernzone nicht wie gewöhnlich nach hinten, sondern nach vorne zu. Die Kerne waren un-regelmässig geordnet und nicht von ovaler Form, sondern stäbchenförmig. Man bekam den Eindruck, als ob die Linsenfasern sich urn ihre Axe umge-dreht hätten. Wie aber eine solche Umdrehung zustande kommen kann, vermag Vortragender nicht zu erklären.

Dr. Snegirew berichtet ferner über einen Fall vom Karzinom des Sinus frontalis. Der sonst gesunde Mann wurde im Jahre 1902 wegen Verdachts auf eine Erkrankung der rechten Stirnhöhle operiert. Bei der Operation wurde eine Geschwulst der vorderen und unteren Wand gefunden. Die Neu-bildung wurde abgetragen, die Höhle ausgekratzt. Glatte Heilung. Erst $1 / 8$ Jahre darauf liess sich der Kranke wieder sehen mit der Angabe, dass seit

M. Monaten sein rechtes Auge ganz erblindet sei und dass er an unerträglichen Kopfschmerzen leide. Die Untersuchung ergab beiderseits Vorwölbung und Schmerzhaftigkeit der vorderen Sinuswand. Bei der Operation erwies sich die rechte Höhle vollständig von Geschwulstmassen ausgefüllt, die bereits in den linken Sinus durchgebrochen waren. An eine radikale Entfernung konnte nicht mehr gedacht werden. Die Operationswunde heilte gut, aber der Zustand des Kranken wurde immer ernster. 2 Monate nach der Operation verliess er das Krankenhaus. Der weitere Schicksal blieb unbekannt. 
